\title{
Poly(anthranilic acid) Nanorods: Synthesis, Characterization and Electrochemical Sensing Properties $†$
}

\author{
R. SURESH ${ }^{\mathrm{a}}$, K. GIRIBABU ${ }^{\mathrm{a}}$, R. MANIGANDAN ${ }^{\mathrm{a}}$, \\ L. VIJAYALAKSHMI ${ }^{\mathrm{b}}$, A. STEPHEN ${ }^{\mathrm{c}}$ and V. NARAYANAN ${ }^{\mathrm{a}}$
}

${ }^{a}$ Department of Inorganic Chemistry, University of Madras, Guindy Campus, Chennai-600 025, India

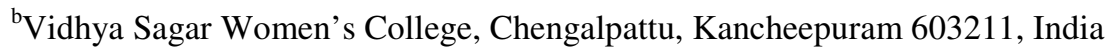

${ }^{\mathrm{c}}$ Department of Nuclear Physics, University of Madras, Guindy Campus, Chennai-600025, India

vnnara@yahoo.co.in

Received 19 January 2013 / Accepted 15 February 2013

\begin{abstract}
Nanostructured poly(anthranilic acid) was synthesized by oxidative polymerization using potassium persulfate as an oxidant and malonic acid (MA) as dopant. The synthesized polymer was characterized by various techniques. The structure of poly(anthranilic acid) was confirmed by FT-IR spectroscopy. The UV-Vis spectra of poly(anthranilic acid) shows $\pi$ - $\pi^{*}$ and inter-ring charge transfer bands. The shift in optical spectra is related to steric repulsion due to the presence of - $\mathrm{COOH}$ groups. The morphological properties were characterized by FESEM which shows the synthesized polymer has rod-like morphology. The electrochemical properties of the poly(anthranilic acid) were studied by cyclic voltammetric method. The synthesized poly(anthranilic acid) nanorods were used to modify the glassy carbon electrode (GCE) and the modified GCE was found to exhibit electrocatalytic activity for the oxidation of dopamine. It shows that the poly(anthranilic acid) will exhibit promising applications in the development of bio-sensors.
\end{abstract}

Keywords: Conducting polymer, Electrocatalyst, Modified electrode, Cyclic voltammetry

\section{Introduction}

Among the conducting polymers, PANI has been the most studied polymer owing to its high conductivity, environmental stability and low cost of production. It has potential application in areas such as sensors ${ }^{1}$, gas separation membranes ${ }^{2}$, light emitting diodes ${ }^{3}$, electrochromic and electronic devices ${ }^{4}$ etc. It can be synthesized conveniently either as a powder or as a film by chemical and electrochemical routes ${ }^{5-6}$. However, one drawback of PANI is its poor processability. PANI itself is not soluble in aqueous and most non-aqueous solvents and it decomposes before it melts. In order to improve its processability, a few authors have reported

$\dagger$ Presented to the National Conference on Chemistry Solutions at SRM University, India 
on the complexation of PANI with sulphonic acids. Alternative way is to use derivatives of PANI like poly(methylaniline), poly(2-methoxyaniline), poly(2-aminophenol) and poly (2-anthranilinc acid) etc. It is believed that the substituent in polymer chain decrease the stiffness of the polymer making it easier to dissolve ${ }^{7}$. In this view, anthranilic acid is an important monomer for the synthesis of carboxylic acid group substituted PANI. Similar to the poly(metanilic acid), it is expected that poly(anthranilic acid), PANA, possesses electrochemical activity over a wide $\mathrm{pH}$ range in aqueous solutions due to the substitution of carboxylic acid group. However, the PANA (with or without external dopant $\mathrm{HCl}$ ) exhibit very low conductivity compared to PANI ${ }^{8}$. In addition, studies on the synthesis of PANA from acidic aqueous solutions are rarely reported in the literature, probably due to its high solubility. It is well known that by using proper dopant, changes the electrical conductivity of the non-conducting or very low conducting polymer to a semiconducting or metallic conducting polymer.

In this paper, we have reported the synthesis of malonic acid doped poly (anthranilic acid) (PANA-MA) nanorods and characterized its structural, optical and electrochemical properties. Additionally we have performed the electrocatalytic activity of the synthesized PANA-MA towards the oxidation of DA.

\section{Experimental}

Anthranilic acid, potassium persulfate, malonic acid, sodium dihydrogen phosphate and disodium hydrogen phosphate were purchased from Qualigens and used without further purification. Dopamine was purchased from Sigma and used as received. Doubly distilled water was used as the solvent.

\section{Synthesis of poly(anthranilic acid)}

In a typical synthesis, $0.69 \mathrm{~g}$ of anthranilic acid and $1.23 \mathrm{~g}$ of malonic acid were dissolved in required amount of double distilled water. The solution was cooled at $5{ }^{\circ} \mathrm{C}$ for $30 \mathrm{~min}$ and then a pre-cooled aqueous solution of potassium persulfate as an oxidant were rapidly added. The molar ratio of oxidant to anthranilic acid in the final solution was $1: 1$. The reaction continued for $16 \mathrm{~h}$ during which the precipitation of black colored poly(anthranilic acid) was observed. The obtained poly(anthranilic acid) was filtered and dried in vacuum at room temperature for $48 \mathrm{~h}$.

\section{Characterization methods}

FT-IR spectrum of the polymer was recorded on Schimadzu FT-IR 8300 series instrument by using potassium bromide pellets. The UV-Visible spectrum of polymer was recorded on UV-1601OC, Shimadzu instrument using absolute ethanol as solvent. The morphology of the polymer was analyzed by FESEM using a HITACHI SU6600 field emission-scanning electron microscopy. The electrochemical experiments were performed on a $\mathrm{CHI} 600 \mathrm{~A}$ electrochemical instrument using the as-modified electrode and bare GCE as working electrode, a platinum wire was the counter electrode and saturated calomel electrode (SCE) was the reference electrode.

\section{Preparation of PANA-MA modified GCE}

Ultrasonic agitation for $30 \mathrm{~min}$ was used to disperse $1 \mathrm{mg}$ of PANA-MA into $5 \mathrm{~mL}$ of water to make blackish brown suspension. $5 \mu \mathrm{L}$ of the PANA-MA suspension was mixed with $10 \mu \mathrm{L}$ of $5 \%$ nafion. The $5 \mu \mathrm{L}$ of the nafion mixed suspension was coated on the polished GCE and dried at room temperature. 


\section{Results and Discussion}

\section{Structure and morphology}

Figure 1 represents the FTIR spectrum of PANA-MA which is in good agreement with previously reported spectrum for PANA ${ }^{9}$. The peak at 1561 and $1514 \mathrm{~cm}^{-1}$ correspond to the $\mathrm{C}=\mathrm{C}$ stretching deformation of quinoid and benzenoid rings respectively. The absorption band at $1692 \mathrm{~cm}^{-1}$ is due to stretching vibration of $\mathrm{C}=\mathrm{O}$ of $-\mathrm{COOH}$ group. The peak at $1383 \mathrm{~cm}^{-1}$ is assigned to the $\mathrm{C}-\mathrm{N}$ stretching of the secondary aromatic amine group. The peak at $1244 \mathrm{~cm}^{-1}$ is commonly attributed to the $\mathrm{C}-\mathrm{N}^{+\bullet}$ stretching vibration in the polaron structure, indicating that the PANA is in the doped state ${ }^{10}$. In addition, the peaks responsible for MA are also observed. The presence of these peaks clearly confirms that MA is incorporated within the PANA.

The UV-Visible spectrum of PANA-MA is shown in Figure 2. The solution spectrum of PANA-MA shows the peak at 525 and $365 \mathrm{~nm}$. The peak observed at $525 \mathrm{~nm}$ corresponds to exciton like transition in quinoiddiimino units. In addition, the other peak that appear at 365 $\mathrm{nm}$ is attributed to $\pi-\pi^{*}$ transition in benzenoid units of the polymer chain. Compared with the same characteristic absorption peak of EB-PANI, it shifts to the longer wavelength (red shift), which indicates that the conjugate conjunction of PANA-MA decreased compared with EB-PANI. It may be due to the resistance effect of - $\mathrm{COOH}$ groups which decreases the planar conjugate conjunction of the aromatic rings ${ }^{11}$.

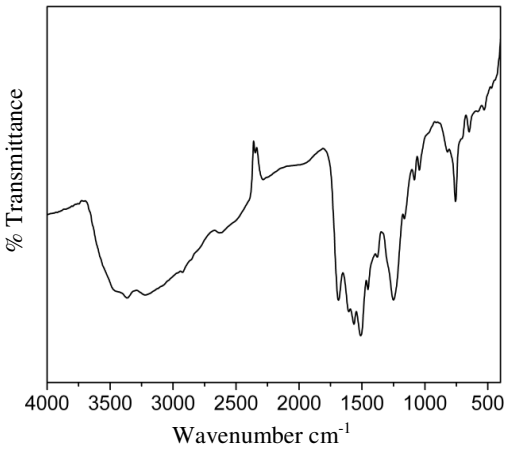

Figure 1. FT-IR spectrum of PANA-MA

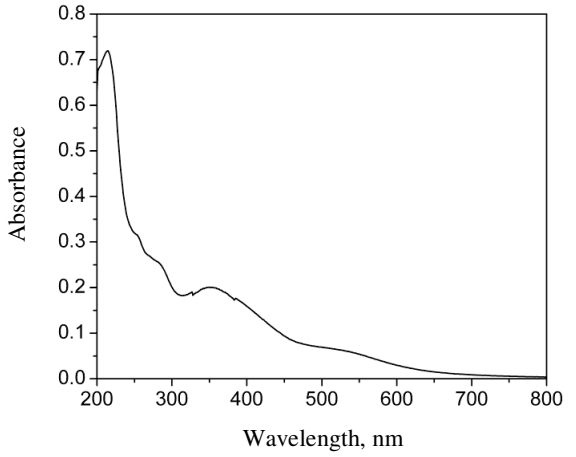

Figure 2. UV-Visible spectrum of PANA-MA

The morphology of the PANA-MA was studied by FESEM which is shown in Figure 3. From the FESEM image, it is clear that the synthesized PANA-MA is rod shaped particles. The length of the nanorod is in the range of 1 to $5 \mu \mathrm{m}$ with the breadth of $100 \mathrm{~nm}$.

\section{Electrochemical property}

The electrochemical behaviour of PANA-MA on GCE was tested through cyclic voltammetric measurements in $0.1 \mathrm{M} \mathrm{HCl}$. Figure 4 depicts the oxidation/reduction process of PANA-MA on GCE. Generally, PANA does not show well-defined redox peaks due to its non-conducting nature. However, PANA-MA shows broad peaks in the potential range from -0.4 to $1 \mathrm{~V}$. Hence, the synthesized PANA-MA is an electroactive polymer which may be due to the dopant, MA. Figure 4 shows a pairs of peaks at $0.284 \mathrm{~V} / 0.109 \mathrm{~V}, 0.573 \mathrm{~V} / 0.453$ $\mathrm{V}$ which are corresponding to emeraldine/pernigraniline and pernigraniline/leucoemaraldine reversible transition. Different scanning rates were used to examine the dynamic property of PANA-MA during the redox process. As is shown in Figure 4, the peak current is directly proportional to the scan rates, suggesting an electric charge-transfer controlled process. 


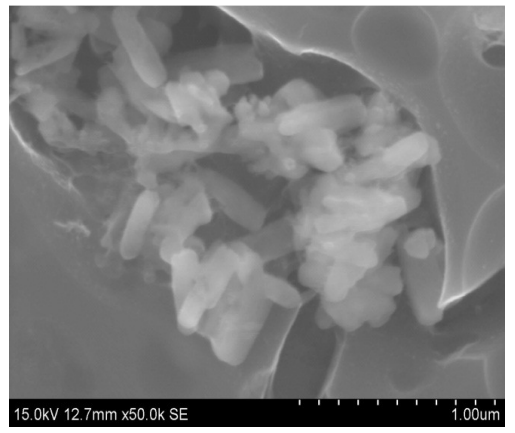

Figure 3. FESEM image of PANA-MA

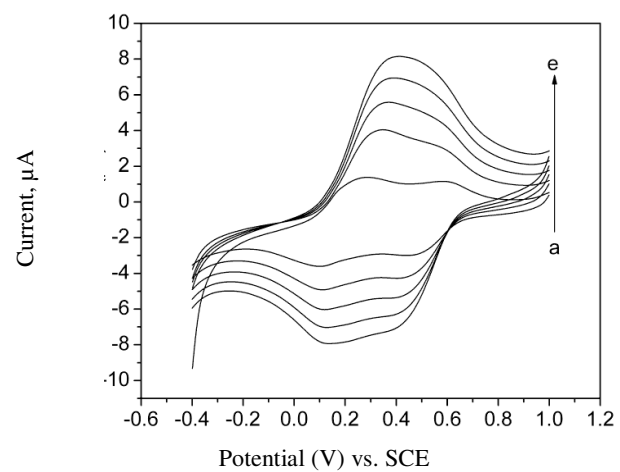

Figure 4. $\mathrm{CV}$ of PANA-MA/GCE at a scan rate of (a) 10 , (b) 20 , (c) 30 , (d) 40 and (e) $50 \mathrm{mV} \mathrm{s}^{-1}$

\section{Electrocatalytic property}

Figure 5 shows the cyclic voltammogram (CV) of $0.5 \mathrm{mM}$ DA at bare and PANA-MA/GCE. The CV of DA (curve a) at the bare GCE shows an oxidation peak at about $+0.49 \mathrm{~V}$. However the DA voltammogram obtained for PANA-MA/GCE showed an oxidation wave with a potential range at $+0.41 \mathrm{~V}$ with enhanced peak current than the bare GCE. Hence, the PANA-MA modified electrode has improved the electron transfer kinetic. The anodic peak potential is shifted to the negative direction and cathodic peak shift towards anodic direction indicates the electrocatalytic ability of the modified electrode.

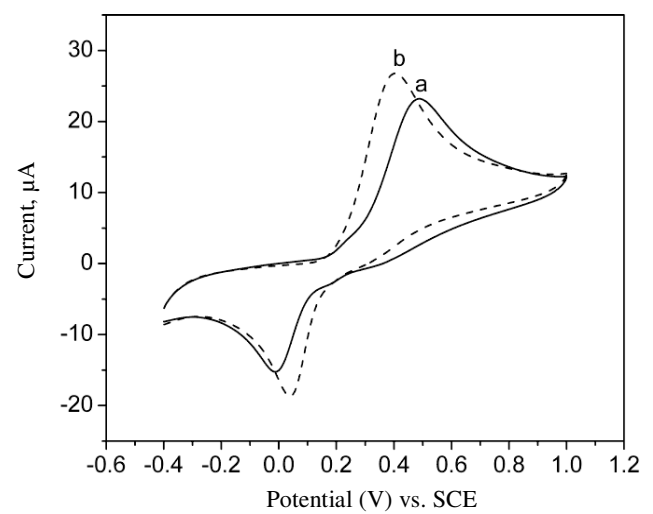

Figure 5. Cyclic voltammogram of $0.5 \mathrm{mM}$ DA (a) bare GCE and (b) PANA-MA/GCE at $50 \mathrm{mV} \mathrm{s}^{-1}$

The effect of scan rate on PANA-MA/GCE in $0.5 \mathrm{mM}$ DA was investigated. The slight shift towards higher values of the oxidation peak potential with increasing scan rates can be observed, indicating a kinetic limitation in the reaction between redox sites of PANAMA/GCE and DA. However, the anodic peak currents for DA at PANA-MA/GC are linearly related to the scan rate in the range of $10-100 \mathrm{mVs}^{-1}$, which indicated that the electron transfer reaction was controlled by adsorption process. In order to determine the adsorption behaviour of UA, we have performed the following experiment. After the PANA-MA/GCE was used for the determination of DA, it was transferred to the blank supporting electrolyte, there was a voltammetric signal for the oxidation of DA appeared and confirming that DA was adsorbed at the PANA-MA/GCE during oxidation 
process. Chang et al. ${ }^{12}$ reported that the UA can form hydrogen bonding with oxo-surface groups, especially $-\mathrm{COOH}$ group in graphene oxide. This adsorption property eliminates the determination of UA form interfering specie like ascorbic acid. In our case, the adsorption of DA at the modified electrode may be due to the presence of - $\mathrm{COOH}$ group in the PANA-MA.

\section{Conclusion}

The MA doped PANA nanorods were synthesized by oxidative polymerization method. The FTIR spectrum confirms the formation of PANA-MA. It also confirms that MA is incorporated within the PANA nanorods. UV-Visible spectra showed the presence of $\mathrm{COOH}$ group in aromatic ring of aniline which produces red shift. Cyclic voltammogram shows that the PANA-MA nanoparticles have electrochemical activity. At pH 7.4 PBS, PANA-MA modified GCE exhibits good electrochemical activity for the oxidation of DA. The modified electrode prepared is very stable and can be utilized for the quantitative determination of DA.

\section{Acknowledgment}

One of the author (RS) acknowledges the CSIR for the financial assistance in the form of Senior Research Fellowship. We acknowledge the FESEM facility provided by the National Centre for Nanoscience and Nanotechnology, University of Madras.

\section{References}

1. Li M, Guo Y, Wei Y, Macdiarmid G A and Lelkes I P, Biomaterials, 2006, 27, 2705.

2. Yang J, Burkinshaw M S, Zhou J, Monkman P A and Brown P J, Adv Mater., 2003, 15, 1081.

3. Karg S, Scott J C, Salem J R and Angelopoulos M, Synth Met., 1996, 80, 111.

4. Alam M M, Wang J, Guo Y, Lee P S and Tseng R H, J Phys Chem B, 2005, 109(26), 12777-12784.

5. Roy B C, Gupta M D, Bhowmilk L and Ray J K, Bull Mater Sci., 2001, 24, 389.

6. Iwuhoa E I, Mavundla S E, Somerset V S, Petrik L F, Klink M J, Sekota M and Baker P, Microchim Acta, 2006, 155, 453-458.

7. Huang L, Wen T and Gopalan A, Mater Lett., 2003, 57, 1765.

8. Chan S H, Ng C S, Sim S W, Tan L K and Tan G T B, Macromolecules, 1992, 25, 6029.

9. Penner R M and Martin C R, J Electrochem Soc., 1986, 133, 310.

10. Zhang L, Peng H, Fang C, Kilmartin P A and Sejdic J T, Nanotecnology, 2007, 18, 115607.

11. Han D, Song J, Ding X, Xu X and Niu L, Mater Chem Phy., 2007, 105, 380.

12. Chang J L, Chang K H, Hu C C, Cheng W L and Zen J M, Electrochem Commun., 2010, 12, 596-599. 